Proc. Indian Acad. Sci. (Earth Planet. Sci.), Vol. 105. No. 1, March 1996. pp. 81-100.

(c) Printed in India.

\title{
Planetary boundary layer over monsoon trough region in a high resolution primitive equation model
}

\author{
K V J POTTY, U C MOHANTY, B NANDI and K J RAMESH \\ Centre for Atmospheric Sciences, Indian Institute of Technology. Hauz Khas, New Delhi \\ 110016, India \\ "Present Affiliation: National Centre for Medium Range Weather Forecasting (NCMRWF), \\ Mausam Bhavan Complex, Lodi Road. New Delhi 110003, India
}

\begin{abstract}
Numerical experiment with improved boundary layer physics has been performed to study the Planetary Boundary Layer (PBL) characteristics over the monsoon trough region. Details of the evolution and structure of the associated boundary layer processes in the monsoon trough and adjoining oceanic regions are examined by integrating the model up to a period of 48 hours.

The model used for this study is a high resolution primitive equation, one with 0.5 latitude/longitude horizontal resolution and 16 levels in the vertical ( 7 levels in the PBL). The boundary layer treatment in the model is based on the Monin-Obukhov similarity theory for the surface layer and Turbulent Kinetic Energy (TKE) formulation based on $E$-s, approach for the mixed layer. The model is integrated using the FGGE level IIIb analysis of European Centre for Medium Range Weather Forecasts (ECMWF), U.K.

The study shows that the diurnal variation of TKE over land is a dominant feature comparing with that over the ocean. Along the monsoon trough region, TKE increases from the eastern end to the western side which is mainly associated with the enhancement of sensible heat flux as we move from the eastern wet land to the western desert sector. It may be noted that the low level wind maximum, which is a characteristic feature over the monsoon region, is well simulated by this improved model physics.
\end{abstract}

Keywords. Planetary boundary layer; turbulent kinetic energy; monsoon trough; energy balance.

\section{Introduction}

The Planetary Boundary Layer (PBL) is that portion of the atmosphere which has close interaction with the underlying earth's surface through the turbulent exchanges of heat, moisture and momentum.

Several observational studies (Palmen 1948; Miller 1958; Tisdale and Clapp 1963; Namias 1973; Gray 1975) and numerical modeling efforts (Anthes and Chang 1978; Krishnamurti et al 1989) have demonstrated the importance of the PBL processes in the development and maintenance of the tropical weather systems. In order to include these PBL processes realistically in a numerical model, it is necessary to parameterize the effects of vertical gradients of heat. moisture and momentum in an appropriate manner.

PBL parameterization involves the representation of the effect of unresolvable sub-grid scale processes into the large scale fields. The effects of PBL can be incorporated in an NWP model broadly in two ways. One way is to parameterize the entire PBL as one layer. The complexity of the single layer PBL parameterization lies in the interdependence of various processes acting in different scales. The second approach 
would be to include several computational levels in the PBL so that the structure of the PBL can be resolved effectively. Further, it requires some type of closure scheme to relate turbulent characteristics with mean quantities. Presently, closure schemes are limited to first, one and a half (TKE) and higher orders. Higher order schemes involve much detailed treatment of PBL physics and numerical complexity, and are hence computationally expensive. First order closure schemes in multi-level models (though computationally economic) are found to be inadequate in resolving the turbulent characteristics realistically (McBean et al 1979). TKE closure is an improvement on the simple first order closure with more physics of the PBL for the estimation of the eddy viscosity coefficient $(K)$.

One dimensional (1-D) multi-level models have been used extensively (Holt and Sethuraman 1988) to understand the PBL characteristics. Due to limitations in incorporating advective and mixing processes, the 1-D models are not so suitable for studying the evolution of PBL and associated characteristics. Hence, the ideal choice would be to have a three-dimensional model having multiple computational levels for resolving PBL characteristics to simulate the PBL effects in the development and maintenance of the tropical weather systems. It is also recognized that increased horizontal resolution together with detailed PBL parameterization would be able to represent the most important characteristics such as moisture convergence and shear zones, which are vital for the development of cumulus convection (Krishnamurti et al 1989) in the tropics.

The monsoon trough boundary layer experiment (MONTBLEX) during 1990 was organized basically to study and simulate the dynamical and thermodynamical processes of PBL over the monsoon trough region. Hence, the Limited Area Model (LAM) of the IIT, Delhi (Mohanty et al 1987) has been modified suitably and implemented over the monsoon trough region with increased vertical resolution close to the surface for the effective treatment of PBL processes, through the incorporation of TKE closure. In this paper, the evolution of the PBL and its characteristics as simulated by the model are studied over the monsoon trough region.

\section{The model}

A high resolution $\left(0.5^{\circ}\right.$ longitude/latitude) hydrostatic, primitive equation model with a terrain following co-ordinate system is used. A brief description of the model is given in table 1. The horizontal domain of the model is from $13.5^{\circ} \mathrm{N}$ to $34.5^{\circ} \mathrm{N}$ in the North-South direction and $66^{\circ} \mathrm{E}$ to $99^{\circ} \mathrm{E}$ in the East-West direction (figure 1). In the vertical, the model involves 16 levels ( 7 levels in the PBL) in sigma $(\sigma)$ co-ordinates. The model uses an envelope topography (mean +1 standard deviation) based on the U.S. Navy high resolution data set.

The continuous governing equations of horizontal momentum, thermodynamic energy, moisture conservation and surface pressure tendency constitute the set of prognostic equations of the model. The hydrostatic and continuity equations are used as diagnostic equations for evaluating geopotential height and vertical motion and thus form a closed system of the primitive equations (PE) of the atmosphere. The prognostic equations include forcing terms, diabatic heating terms and source/sink terms, which are regarded as physical processes and are treated separately. The primitive equations are written in flux form involving sigma co-ordinates. The Arakawa 
Table 1. An overview of the model.

\section{Domain}

Independent variables

Prognostic variables

Diagnostic variables

Topography

Vertical grid system

Vertical levels of the model

Horizontal grid system

Space finite difference scheme

Time integration scheme

Time step

$\Delta \dot{\lambda}, \Delta \phi$

Horizontal diffusion

Initialisation

Physical processes $66^{\circ}-99^{\circ} \mathrm{E} ; 13.5^{\circ} \mathrm{N}-34.5^{\circ} \mathrm{N}$.

$i, \phi, \sigma, t$.

$u, v, T, q, P s$.

$\phi, \dot{\sigma}$.

Envelope (mean +1 std)

16 levels (sigma $(\sigma)$ co-ordinates).

$0.05,0.15,0.25,0.35,0.45,0.55$

$0.65,0.75,0.82,0.86,0.90,0.935$, $0.960,0.975,0.987$ and 0.997 .

: Arakawa C-grid.

: Second order accuracy.

: Split-explicit time integration.

: $\Delta t=150$ seconds

: $\quad 0.5$ Lat./Long. $(50 \mathrm{~km})$.

: Linear second order.

: Non-linear normal mode.

: Dry convective adjustment; deep cumulus convection 'Kuo Scheme' (Anthes 1977) with convective precipitation.

: Large scale stratified precipitation with $\mathrm{RH}>95 \%$.

PBL; M-O similarity approach for the surface layer, $\operatorname{TKE}(E-\varepsilon)$ for the mixed layer.

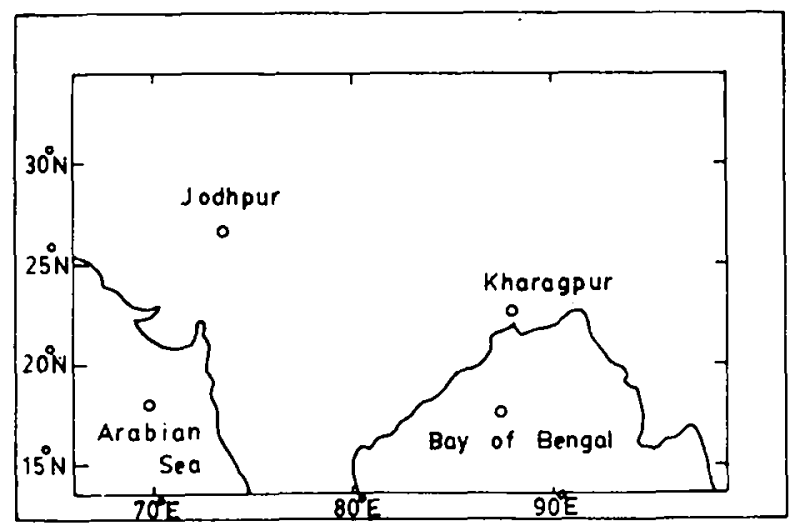

Figure 1. Model domain and the grid points chosen for the study.

C-grid in spherical co-ordinates is used for horizontal differencing. Time dependent lateral boundary conditions of Perkey and Kreitzberg (1976) are used at the boundary points. A second order diffusion is used in the model to suppress the computational instabilities. The time integration scheme implemented in the model is the split-explicit 
method which allows larger time steps by effectively separating various terms of the prognostic equations into parts governing the slow moving Rossby and fast moving gravity waves.

Model physics includes parameterizations of PBL (TKE: $E-\varepsilon$ ), cumulus convection (Kuo 1974), large scale precipitation and dry convective adjustment.

Details of the model equations and numerics may be obtained from Mohanty et al (1987) and Madala et al (1987).

\section{PBL parameterization scheme of the model}

Surface temperature plays an important role in the evolution of PBL processes and hence the prediction of ground temperature is also included in the surface layer for grid points over the land.

\subsection{Ground temperature}

The ground temperature $\left(T_{g}\right)$ is predicted with the help of surface energy balance equation (Chang 1979):

$$
\frac{\delta T_{g}}{\delta t}=R C_{g}^{-1}+W\left(T_{m}-T_{g}\right)+\rho C_{p}\left(\overline{w^{\prime} \theta^{\prime}}\right)_{0} C_{g}^{-1} .
$$

The first term of the right hand side (r.h.s) represents the net radiation, the second term represents the heat exchange with the deep soil and the last term the heat exchange with the overlying atmosphere. Here $R$ is the net radiation, $C_{g}$ is the heat capacity per unit area of the soil, $W$ is the inverse of the time scale for the heat conduction in the soil ( $2 \pi / 1$ day), $T_{m}$ is the deep soil temperature. In the last term of equation (1), $\rho$ is the air density. $C_{p}$ the specific heat at constant pressure and $\overline{u^{\prime} \theta^{\prime}}$ the potential temperature flux. The subscript 0 denotes the near surface value.

Net radiation $R$ is given by,

$$
\begin{aligned}
& R=I_{S}+I_{L}-Q_{L}, \\
& I_{S}=\text { Incoming solar radiation observed at the surface, } \\
& I_{L}=\text { Atmospheric long wave back scattering radiation, } \\
& Q_{L}=\text { Outgoing long wave radiation. }
\end{aligned}
$$

$I_{S}$ is given by.

$$
I_{S}=S \cos z(1-A) b^{\sec z} .
$$

where $S$ is the solar constant, $A$ is the surface albedo and $b$ is the atmospheric turbidity, which is a function of precipitation.

The zenith angle $z$ is obtained from the local hour angle $h \alpha$. latitude $\phi$ and declination $\delta$,

$$
\cos z=\cosh \alpha \cos \phi \cos \dot{\delta}+\sin \phi \sin \dot{\delta} .
$$


Water vapour is ignored in the relationship for net long wave radiation computed using Brunt's equation (Seller 1965),

$$
I_{L}-Q_{L}=\alpha(1-0.61) \beta T_{g}^{-4}
$$

where $\alpha$ is the soil emissivity which is a function of precipitation and $\beta$ is the Stefan-Boltzmann constant. Heat capacity of soil slab $C_{8}$ is approximated as an $80 \mathrm{~m}$ deep air layer (Chang 1979). Oceanic grid points use the climatologically averaged sea surface temperature as $T_{g}$.

\subsection{Surface layer}

The surface boundary layer (constant flux layer) is parameterized based on the similarity theory (Monin and Yaglom 1971), using similarity functions (Businger et al 1971),

$$
\begin{aligned}
& \frac{k z}{U_{*}} \frac{\partial u}{\partial z}=\phi_{m}(z / L), \\
& \frac{k z}{\theta_{*}} \frac{\partial \theta}{\partial z}=\phi_{h}(z / L) \\
& \frac{k z}{q_{*}} \frac{\partial q}{\partial z}=\phi_{h}(z / L)
\end{aligned}
$$

where the non-dimensional stability parameters $\phi_{m}$ and $\phi_{h}$ are functions of the MoninObukhov length $L$ and $k$ is Von-Karman constant.

\subsection{Mixed layer}

Treatment of the mixed layer in TKE closure models involves two types of approaches for the evaluation of turbulent dissipation of kinetic energy $(\varepsilon)$. The first method is based on mixing length approach $(E-l)$ and the second approach $(E-\varepsilon)$ involves the time tendency equation for $\varepsilon$ evaluation. It was found in the studies of Holt and Sethuraman (1988) using MONEX data sets that TKE closure based on $E-\varepsilon$ formulation performs better than the $E-l$ approach. Therefore in this study the PBL is parameterized by the TKE: $E-\varepsilon$ closure with the constants of Detering and Etling (1985).

The prognostic equation for turbulent kinetic energy (TKE) is derived as (Monin and Yaglom 1971),

$$
\frac{\partial E}{\partial t}=-\overline{u^{\prime} w^{\prime}} \frac{\partial u}{\partial z}-\overline{v^{\prime} w^{\prime}} \frac{\partial v}{\partial z}+\frac{g}{\theta} \overline{w^{\prime} \theta^{\prime}}-\frac{\partial}{\partial z}\left(\overline{w^{\prime} E^{\prime}}+\overline{\frac{p^{\prime} w^{\prime}}{\rho}}\right)-\varepsilon
$$

First two terms on the right hand side represent the shear production, third represents the buoyancy production, the fourth turbulent transport of kinetic energy and pressure, and the fifth dissipation of turbulent kinetic energy. In the above equation several terms have to be parameterized. 
Shear terms are related to the vertical gradient of the mean wind by eddy viscosity coefficient $K_{m}$ which is a property of the turbulent flow,

$$
-\overline{u^{\prime} w^{\prime}} \frac{\partial u}{\partial z}-\overline{v^{\prime} w^{\prime}} \frac{\partial v}{\partial z}=K_{m}\left[\left(\frac{\partial u}{\partial z}\right)^{2}+\left(\frac{\partial v}{\partial z}\right)^{2}\right]
$$

similar assumption is valid for heat flux $\left(\overline{w^{\prime} \theta^{\prime}}\right)$ in the buoyancy term and is parameterized as (Deardorff 1966),

$$
-\frac{g}{\theta} \overline{w^{\prime} \theta^{\prime}}=\frac{g}{\theta} K_{h}(\partial \theta / \partial z)
$$

The kinetic energy and pressure terms which transport stresses from one place to another are small near the surface but become prominent away from the surface. These terms are treated together and assumed to be of the gradient diffusion type (Shir 1973),

$$
-\left(\overline{w^{\prime} E^{\prime}}+\overline{p^{\prime} w^{\prime}} / \rho\right)=C_{1}\left(K_{m} \frac{\partial E}{\partial z}\right)
$$

where $C_{1}$ is a constant.

Substitution of these parameterizations into (9) yields,

$$
\frac{\partial E}{\partial t}=K_{m}\left[\left(\frac{\partial u}{\partial z}\right)^{2}+\left(\frac{\partial v}{\partial z}\right)^{2}\right]+\frac{g}{\theta} K_{h} \frac{\partial \theta}{\partial z}+C_{1} \frac{\partial}{\partial z}\left(K_{m} \frac{\partial E}{\partial z}\right)-\varepsilon
$$

The prognostic equation for the dissipation of TKE is given by

$$
\frac{\partial \varepsilon}{\partial t}=C_{3} \frac{\varepsilon}{E}\left(-\overline{u^{\prime} w^{\prime}} \frac{\partial u}{\partial z}-\overline{v^{\prime} w^{\prime}} \frac{\partial v}{\partial z}+\frac{g}{\theta} \overline{w \theta^{\prime}}\right)-C_{4} \frac{\varepsilon^{2}}{E}+C_{5} \frac{\partial}{\partial z}\left(K_{m} \frac{\partial \varepsilon}{\partial z}\right)
$$

The first term on the r.h.s of equation (14) represents the production, second term destruction, and the last term vertical transport of dissipation.

The relationship of eddy viscosity $\left(K_{m}\right)$ and TKE in terms of $\varepsilon$ is given by (Daly and Harlow 1970)

$$
K_{m}=C_{2} E^{2} / \varepsilon
$$

Holt and Sethuraman (1988) have studied the suitability of the values of constants used in the above equations of TKE and dissipation and found that constants of Detering and Etling (1985) suit better. Hence for the present study we used the same constants, which are given by

$$
C_{1}=1 \cdot 35, C_{2}=0.026, C_{3}=1 \cdot 13, C_{4}=1.9 \text { and } C_{5}=0.77 \text {. }
$$

Solution of turbulent kinetic energy equation requires the specification of $E$ in the surface layer (Mailhot and Benoit 1982), which should be a function of $u_{*}, w_{*}$ and $z / L$. Based on Deardorff (1974b) and Wyngaard (1975), who give

$$
\begin{array}{ll}
E=3.75 U_{*}^{2} & z / L>0 \\
E=3.75 U_{*}^{2}+0.2 \mathrm{~W}_{*}^{2}+(-z / L)^{2 / 3} U_{*}^{2} & z / L<0 \\
\varepsilon=U_{*}^{3} / k z &
\end{array}
$$


where $U_{*}$ is the frictional velocity and $W_{*}$ is the convective velocity

$$
W_{*}=\left[(g / T) h\left(\overline{w^{\prime} T_{i}^{\prime}}\right)_{0}\right]^{1 / 3}
$$

the subscript zero denotes near-surface values and $T_{r}$ the virtual temperature. The flux $\overline{w^{\prime} T_{\imath}}$, is computed as

$$
\overline{w^{\prime} T_{v^{\prime}}}=\overline{w^{\prime} T^{\prime}}+0.61 \overline{T^{\prime} w^{\prime} q^{\prime}}
$$

where $g$ is the acceleration due to gravity and $h$ is the boundary layer height, which is computed from the TKE of the previous iteration. $h$ is given as the model level height at which TKE reduces to $0.05 \mathrm{~m}^{2} / \mathrm{s}^{2}$ or less. Upper boundary condition is taken as $E=\varepsilon=0$.

The convective velocity scale $W_{*}$, which is a measure of the variance of the horizontal velocity components, has been included in the above equation for unstable case to reflect the horizontal motions induced in the lower boundary layer by the vertical motion in the large eddies of the upper boundary layer. The $z / L$ term gives the dependence of $\overline{w^{\prime 2}}$ on $z$.

\section{Data}

FGGE level III-b analysis produced at ECMWF, U.K. for the period from 5 th -7 th July 1979 at 1200 UTC has been used. U.S. Navy topography data and climatological sea surface temperature (SST) for July have also been used for the model simulation.

Among the precipitating systems in the monsoon period, the monsoon depression is a major synoptic system. On 5th July 1979 at 1200 UTC a low pressure area was present over north-east Bay of Bengal centred close to $20^{\circ} \mathrm{N}$ and $90^{\circ} \mathrm{E}$, intensified into a depression in 24 hours and further intensified into a deep depression after 48 hours. With the presence of low/depression over northern part of Bay of Bengal, normal monsoon conditions prevailed over the country.

\section{Experiment}

The objective of the experiment is to study the evolution of the PBL structure over the monsoon trough region. Development of PBL at any surface is determined by thermal and dynamical effects locally. During day time, the land surface gets heated up at a much faster rate than the oceanic surface resulting in a large diurnal variation of surface temperature and surface characteristics. As the western sector of the monsoon trough falls under the dry convective regime, the evolution of the PBL and associated characteristic features are largely controlled by the thermal effects alone. On the other hand, the eastern sector of the monsoon trough is categorized as a moist convective region and hence the turbulent transfer of sensible heat and its diurnal variation is considerably less. At the same time, the local dynamical effects play a significant role in the evolution of PBL over the eastern side of the monsoon trough. Hence we have chosen two grid points of the model close to the stations Jodhpur and Kharagpur representing the western and eastern sectors of the monsoon trough respectively and 
one each over the Arabian Sea and Bay of Bengal to study the evolution of the PBL and the associated features over the Indian sub-continent and adjoining oceanic sectors through the incorporation of improved PBL physics in a high resolution LAM (figure 1). The model has been integrated up to 48 hours starting from 1200 UTC on 5th July 1979. Since our aim is to study the PBL characteristics, the discussion of the results is restricted largely up to 24 hours of model simulation.

\section{Results and discussion}

In this section, results of $\mathrm{PBL}$ characteristic features over the monsoon trough region and adjoining oceanic areas as well as simulation of large scale fields are discussed.

\subsection{PBL characteristic features}

Evaluation of the PBL at various selected points of the model domain is studied through the analysis of the variation of Turbulent Kinetic Energy (TKE) as it is considered as a measure of turbulence intensity. Figure 2 depicts the variation of TKE with height at every hour of the model integration. It is found that, in general, evolution of TKE from day to night conditions over the land points is the most dominant feature. Due to the absence of diurnal variation of surface temperature over the sea points, no significant variations in the TKE magnitudes are observed. Accordingly, the vertical extent of TKE is found to be minimum over the sea points all through the 24 hours of integration. Over the land. TKE magnitudes are found to be more during the evening time (1300-1700 hrs of IST), and the comparison of the relative magnitudes shows that TKE is maximum over the western part of the monsoon trough (Jodhpur) and minimum over the eastern end (Kharagpur) and the diurnal variation of TKE is found to be minimum over the eastern side of the monsoon trough in comparison with the western end. The appearance of maximum TKE magnitudes over the western end of the trough (Jodhpur) could be due to the intense heating during the day followed by cooling in the night. In contrast, over the eastern part of the trough, abundant cloud cover restricts the amount of solar insolation reaching the ground and moist land surface with large evaporation from ground leads to reduced diurnal variation of TKE.

The vertical profiles of TKE (figure 3 ) and eddy viscosity coefficient $\left(K_{m}\right)$ (figure 3 ) depicted at 6-hour intervals of the model integration are analysed to study the evolution of PBL characteristics at various selected points of the domain. The profiles of TKE show that TKE values are extremely small at $t+6$ hours ( $23.30 \mathrm{hrs}$ of IST) having maximum values close to the ground. With the increase in the intensity of turbulence, the magnitude of TKE increases generally and the level at which maximum TKE is observed moves to higher altitudes. Here again, the maximum value of TKE at $t+24 \mathrm{hrs}$ is found to be more at Jodhpur $\left(1.3 \mathrm{~m}^{2} / \mathrm{s}^{2}\right)$ as compared to that of Kharagpur $\left(0.6 \mathrm{~m}^{2} / \mathrm{s}^{2}\right)$. At Jodhpur, the TKE is found to be more at $t+12 \mathrm{hrs}(05.30 \mathrm{hrs}$ IST) as compared to $t+18 \mathrm{hrs}(11.30 \mathrm{hrs}$ IST). This could be attributed to the presence of a secondary maximum at this time appearing mainly due to the wind shear. Among the sea points considered, the magnitudes of TKE are found to be more over the Arabian Sea due to the presence of more wind shear over this region than that at the Bay of Bengal. Similar variations of $K_{m}$ (figure 3 ) are found at various selected points of the domain. 

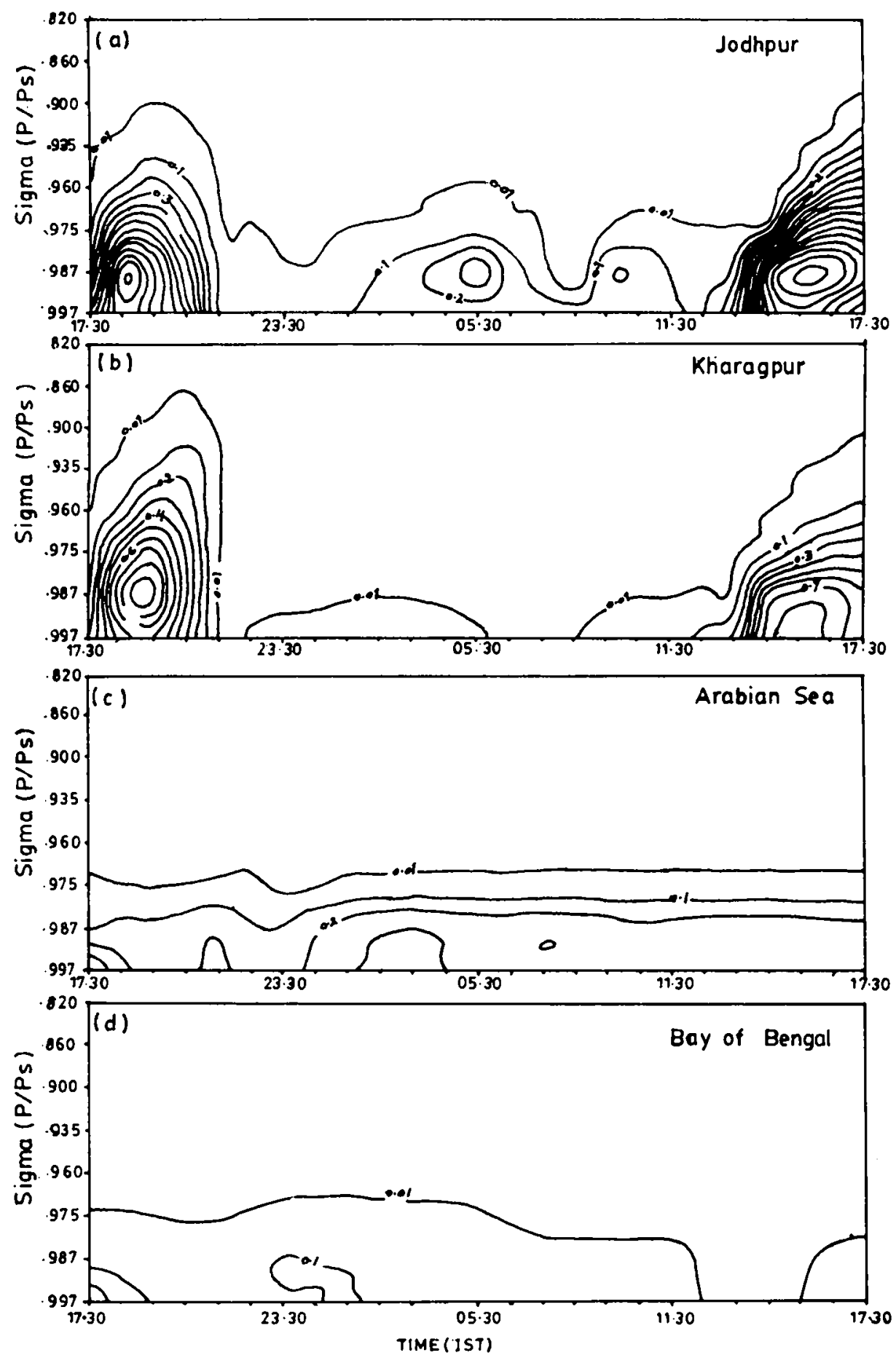

Figure 2. Diurnal varration of TKE $\left(\mathrm{m}^{2} / \mathrm{s}^{2}\right)$ with height: (a) Jodhpur: (b) Kharagpur: (c) Arabian Sea: (d) Bay of Bengal. 
The height of the planetary boundary layer is an important parameter for the evolution of TKE. In order to assess the planetary boundary layer height, observed virtual potential temperature profile of Calcutta and Jodhpur has been compared with the model simulated profile (figure 4). Observed boundary layer height shows good agreement with the simulated one at Calcutta. But at Jodhpur, the inversion layer is higher than that observed on 6th July, 1979 at 1200 UTC.

The hourly rainfall plots at the four selected points of the domain are illustrated in figure 5. The rainfall variation does not show any significant influence over the diurnal variation of TKE as no explicit treatment of water droplets is included in the PBL parameterization. Due to the dry convective type of PBL over Jodhpur, convective rainfall is not predicted. But over the eastern end of the monsoon trough (Kharagpur) and in the Bay of Bengal due to formation of a depression over the head of the Bay (by $12 Z$ of 6 th July 1979), certain rainfall amounts are predicted by the model. On the other
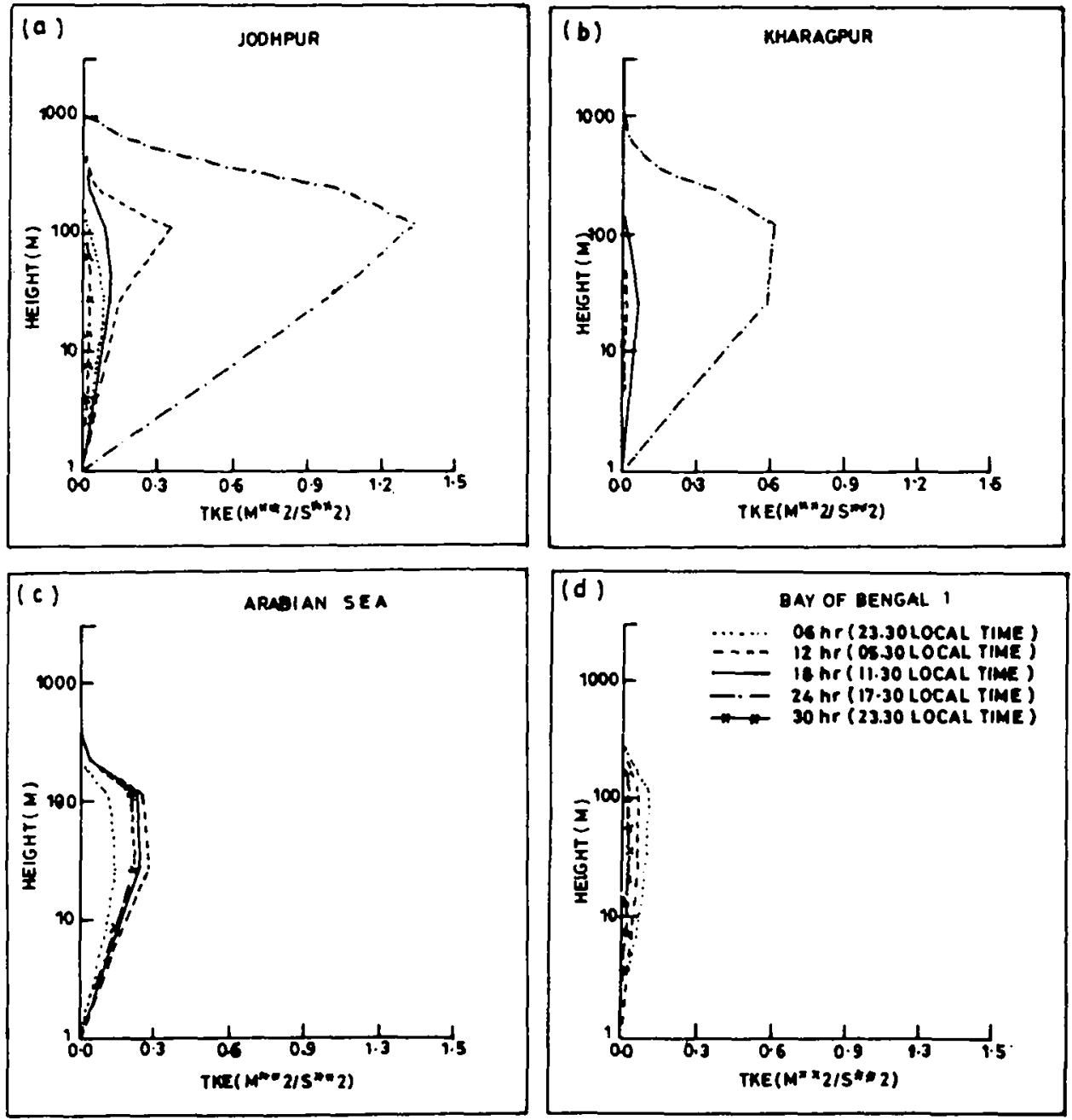

Figure 3. (Continued) 

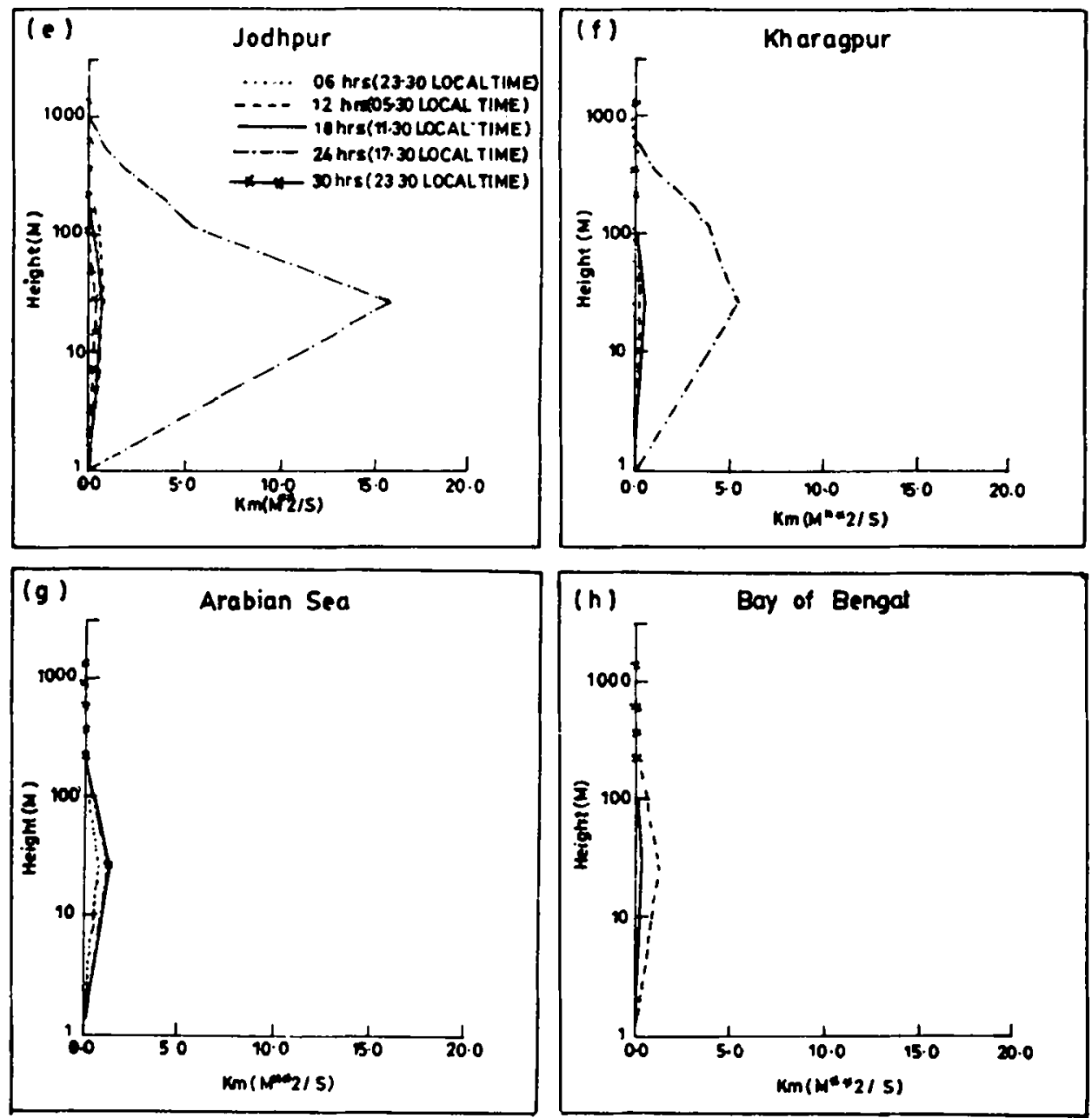

Figure 3. Vertical profiles of TKE and eddy viscosity $\left(K_{m}\right)$ at 6 hours of interval. TKE: (a) Jodhpur; (b) Kharagpur; (c) Arabian Sea; (d) Bay of Bengal.

Eddy viscosity: (e) Jodhpur; (f) Kharagpur; (g) Arabian Sea; (h) Bay of Bengal.

hand, rainfall over the Arabian Sea is mainly attributed to the local convective processes.

Latitudinal mean vertical cross-section of wind and temperature fields is analysed over the monsoon trough region to identify the characteristic features of PBL generated by the model physics. Domain of $70^{\circ} \mathrm{E}-90^{\circ} \mathrm{E}$ and $22^{\circ} \mathrm{N}-27^{\circ} \mathrm{N}$ is chosen, which usually covers the normal monsoon trough region and dominantly covers the land region. Vertical cross-sections of wind and temperature at initial time $(t=0)$ and after $24 \mathrm{hrs}$ of the model integration are shown in figure 6 . At the starting time of the model integration (figure 6a) the wind field distribution does not display any variation all along the trough region in the lower-most levels. However, at $0.85 \sigma$ approximately, the wind is found to be maximum over the western end of the trough as compared to the eastern end. After the integration of the model for $24 \mathrm{hrs}$ (figure $6 \mathrm{~b}$ ), the model could 

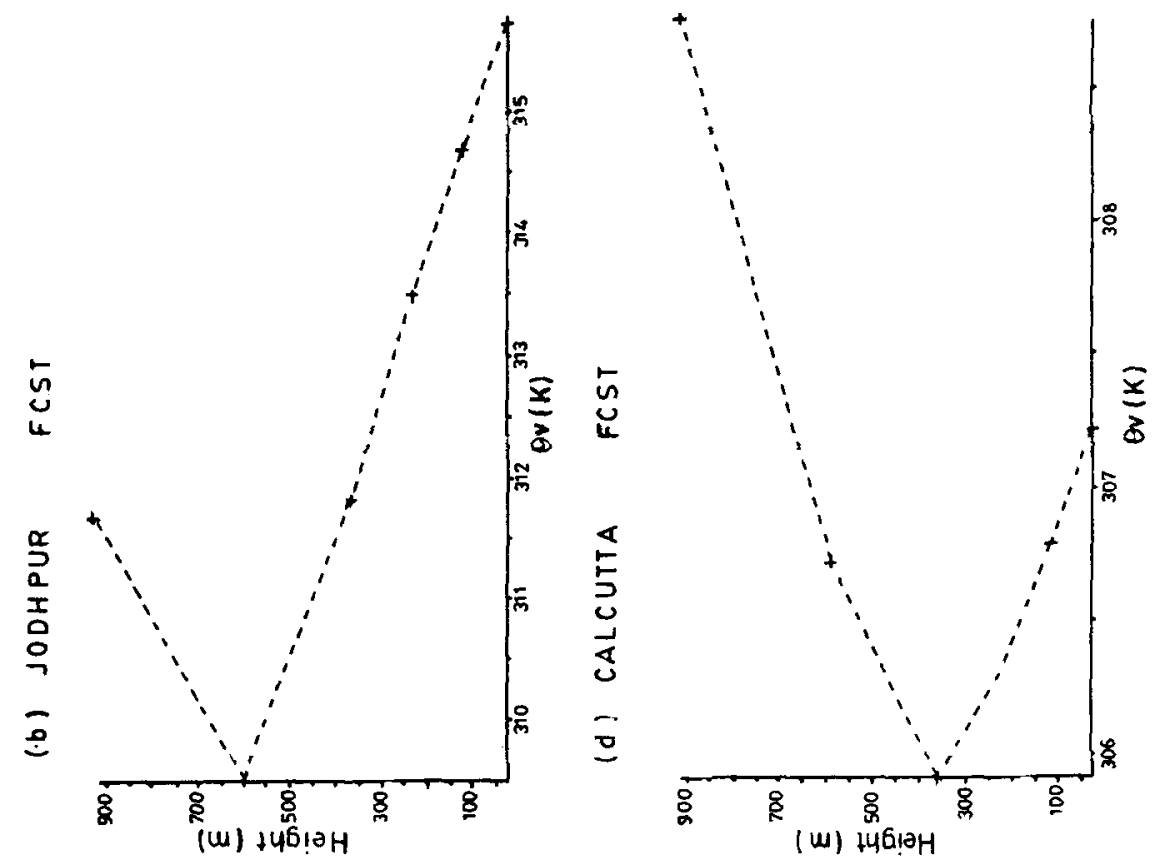

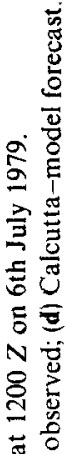
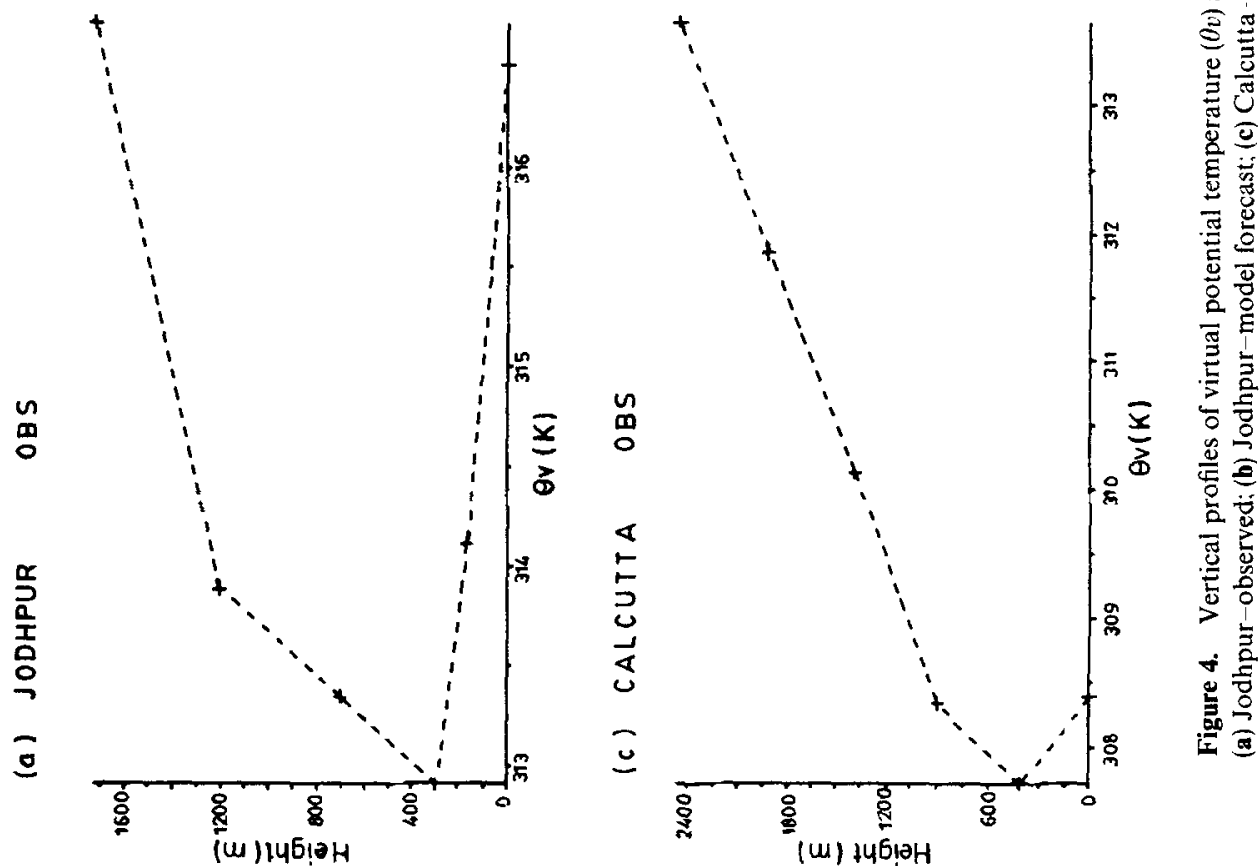

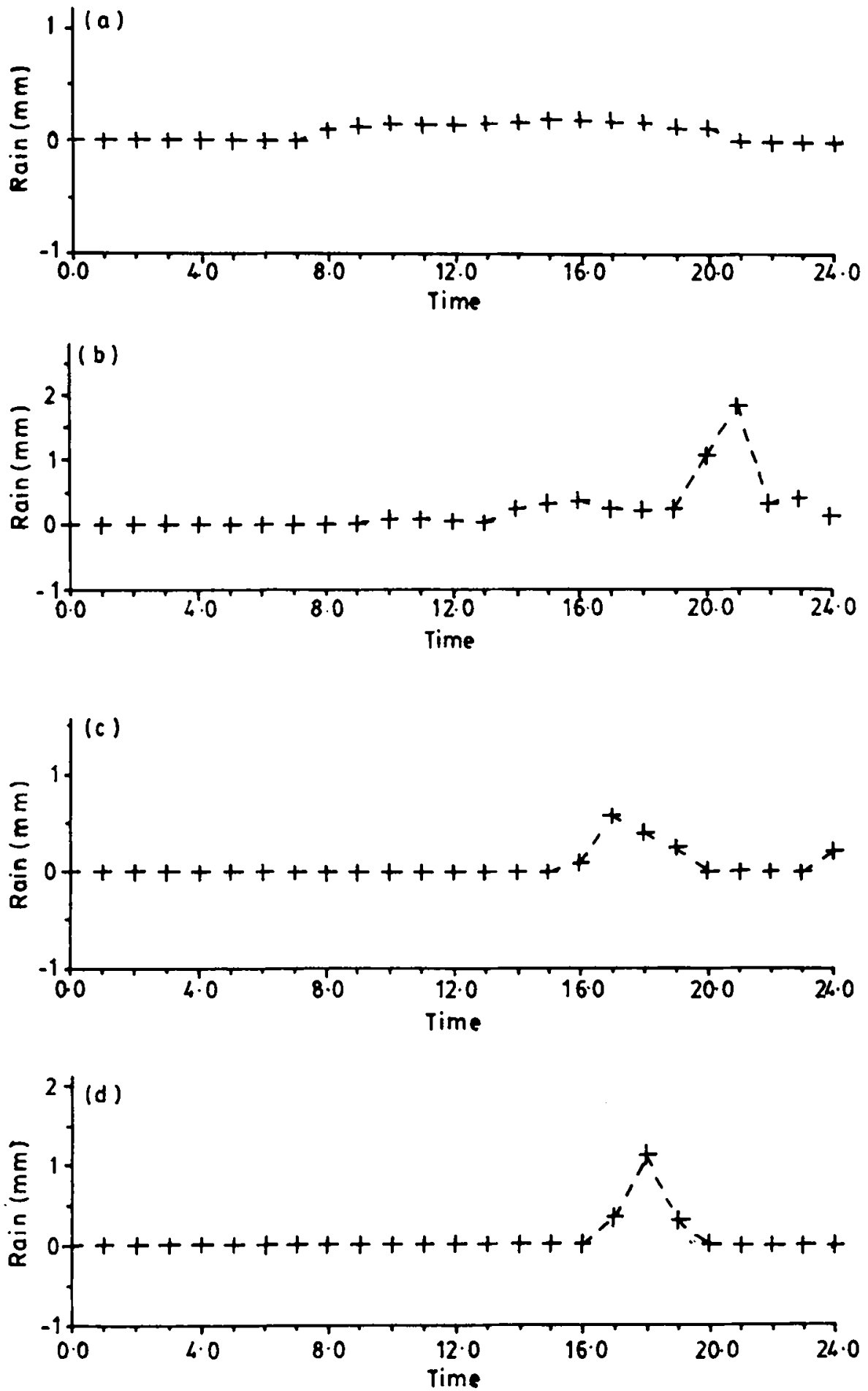

Figure 5. Hourly rainfall.

(a) Jodhpur; (b) Kharagpur; (c) Arabian Sea; (d) Bay of Bengal. 

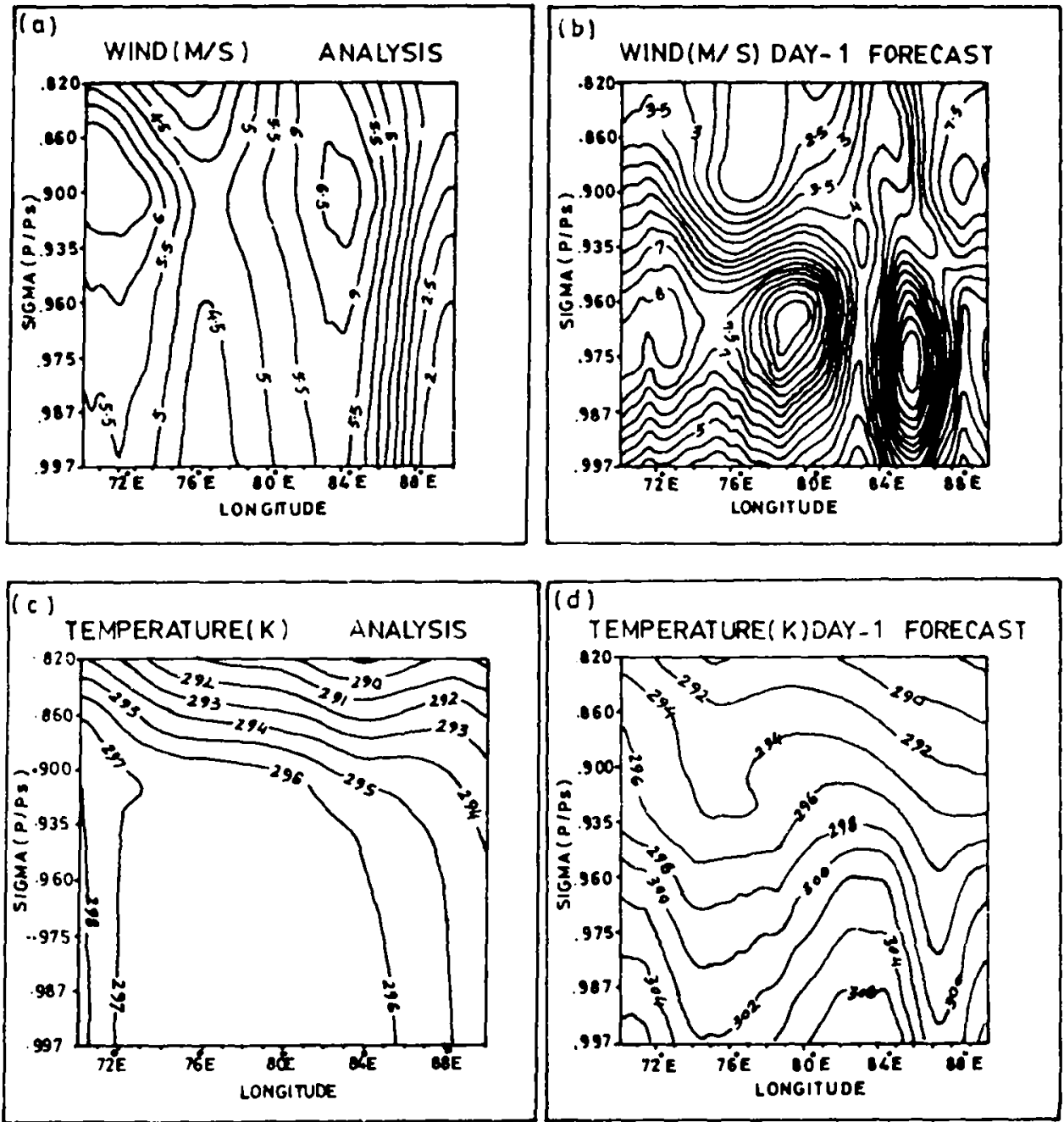

Figure 6. Zonally averaged fields of wind and temperature.

(a) Analysis wind; (b) Forecast wind; (c) Analysis temperature; (d) Forecast temperature.

simulate the low level wind maxima which is a characteristic feature of a turbulent boundary layer. The appearance of wind maxima over the monsoon trough region is also observed in the observational studies (Tyagi et al 1994) under the strong and active monsoon conditions. Usually analysis is done for $1000 \mathrm{mb}$ and $850 \mathrm{mb}$ and hence the maxima are seen at about $0.9-0.85 \sigma$ level in the analysis field. Since the model has a much higher vertical resolution close to the surface (seven levels), in the simulation the low level wind maxima are found in the column $(0.96-0.987 \sigma)$ closer to the surface. Hence, such a feature cannot be resolved from the analysed data sets. The existence of such a feature close to the surface $(0.15-0.6 \mathrm{~km})$ is also observed at $12 \mathrm{Z}$ on 6th July 1979 over Ranchi, Lucknow, Delhi, Gwalior and Jodhpur stations situated along the monsoon trough (figure 7). It is expected that the analysis of the MONTBLEX-90 data sets would be able to throw some light on the observed structure of PBL in a much 

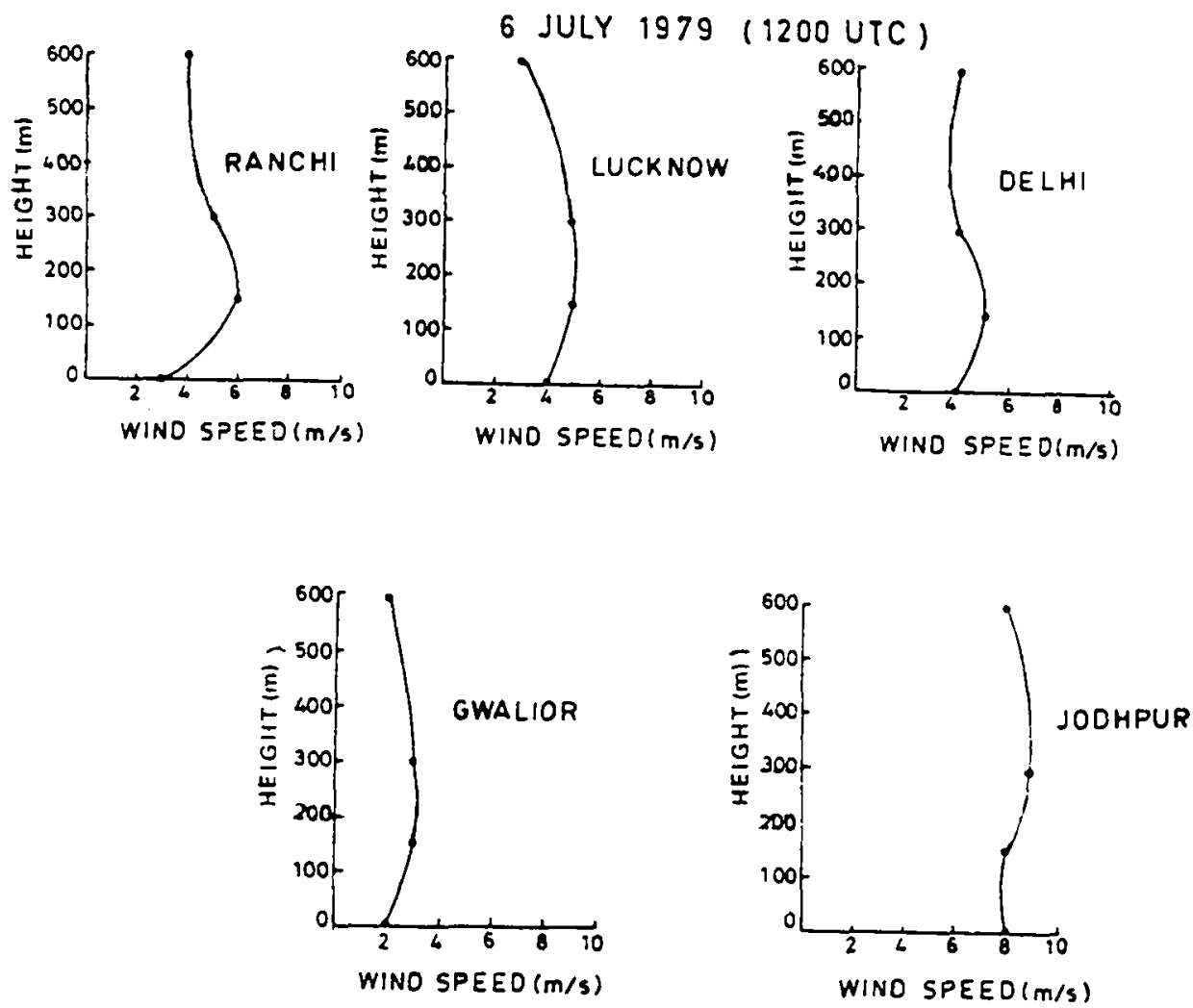

Figure 7. Analysis of wind at $1200 Z$ on 6th July 1979.

more detailed manner. It is also interesting to note that the level of wind maxima increases from east to west across the monsoon trough region.

In accordance with the increase of turbulence intensity all along the monsoon trough region as seen earlier, the stable atmospheric conditions prevailing over the central parts of the trough (figure $6 \mathrm{c}$ ) at $t=0$ transformed into an unstable regime as observed at $t+24 \mathrm{hrs}$ of the model integration (figure 6d). Both of these characteristics have been brought out by the model simulation as stated above, as it is apparent that such a feature was not existing at the beginning of the model integration.

\subsection{Large scale fields}

There was a developing vortex on 5th July 1979 over the north Bay of Bengal and its further developments can be seen in the prediction fields (figure 8 ) as well as in the verifying analyses (figure 9). The monsoon trough is well-marked to the east of $80^{\circ} \mathrm{E}$ from central India to the north Bay of Bengal without any significant slope with height. Wind flow analysed at $0.9 \sigma$ surface indicates that the vortex and associated circulation features over the north Bay of Bengal has been captured reasonably well by the model. Though the predicted center of the vortex lies to the east of its location (about $0.5^{\circ}$ 


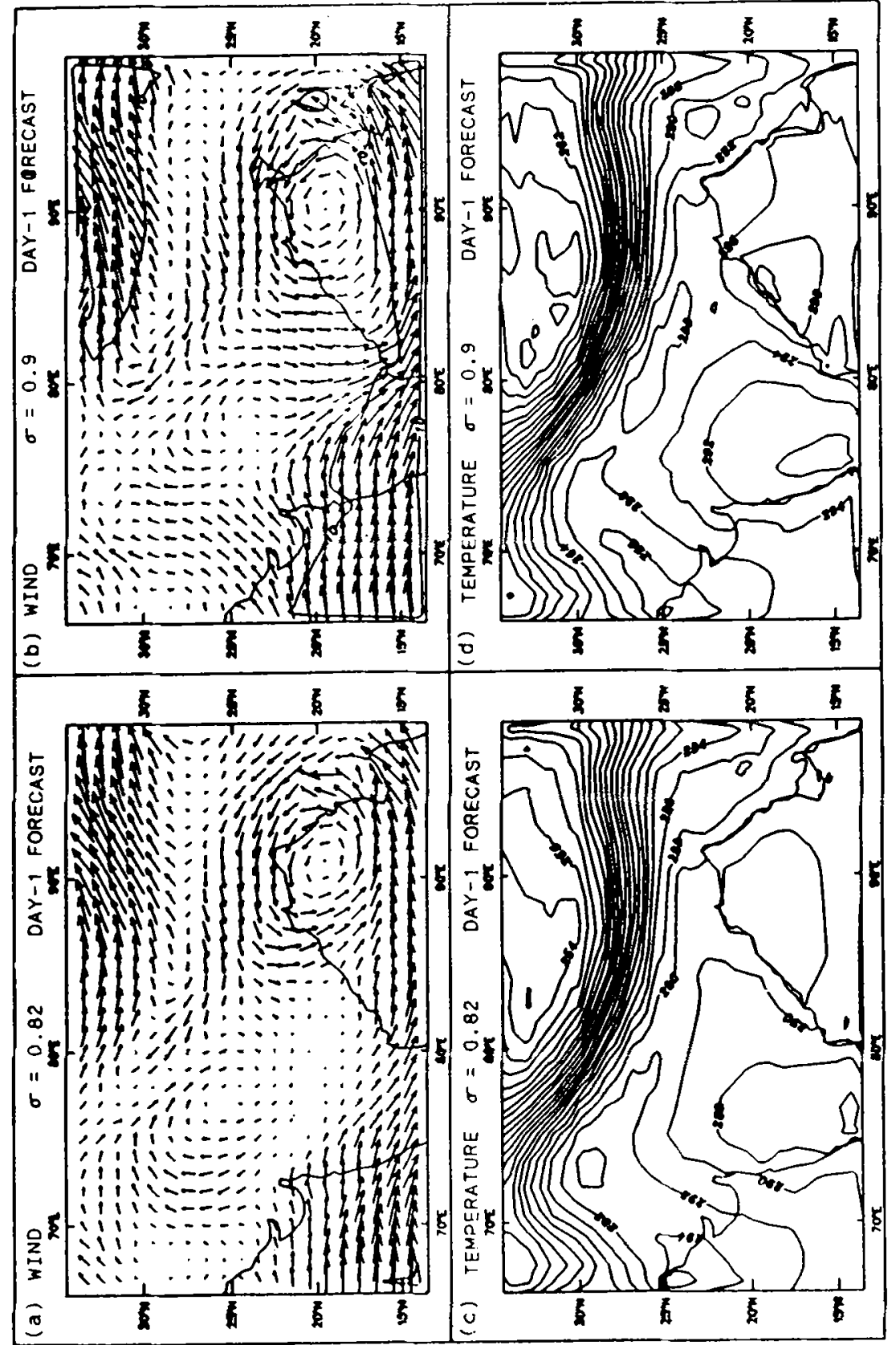

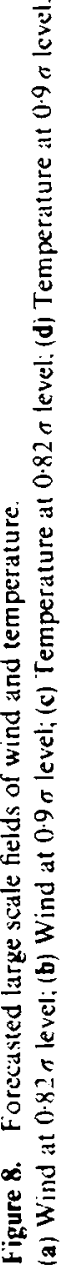




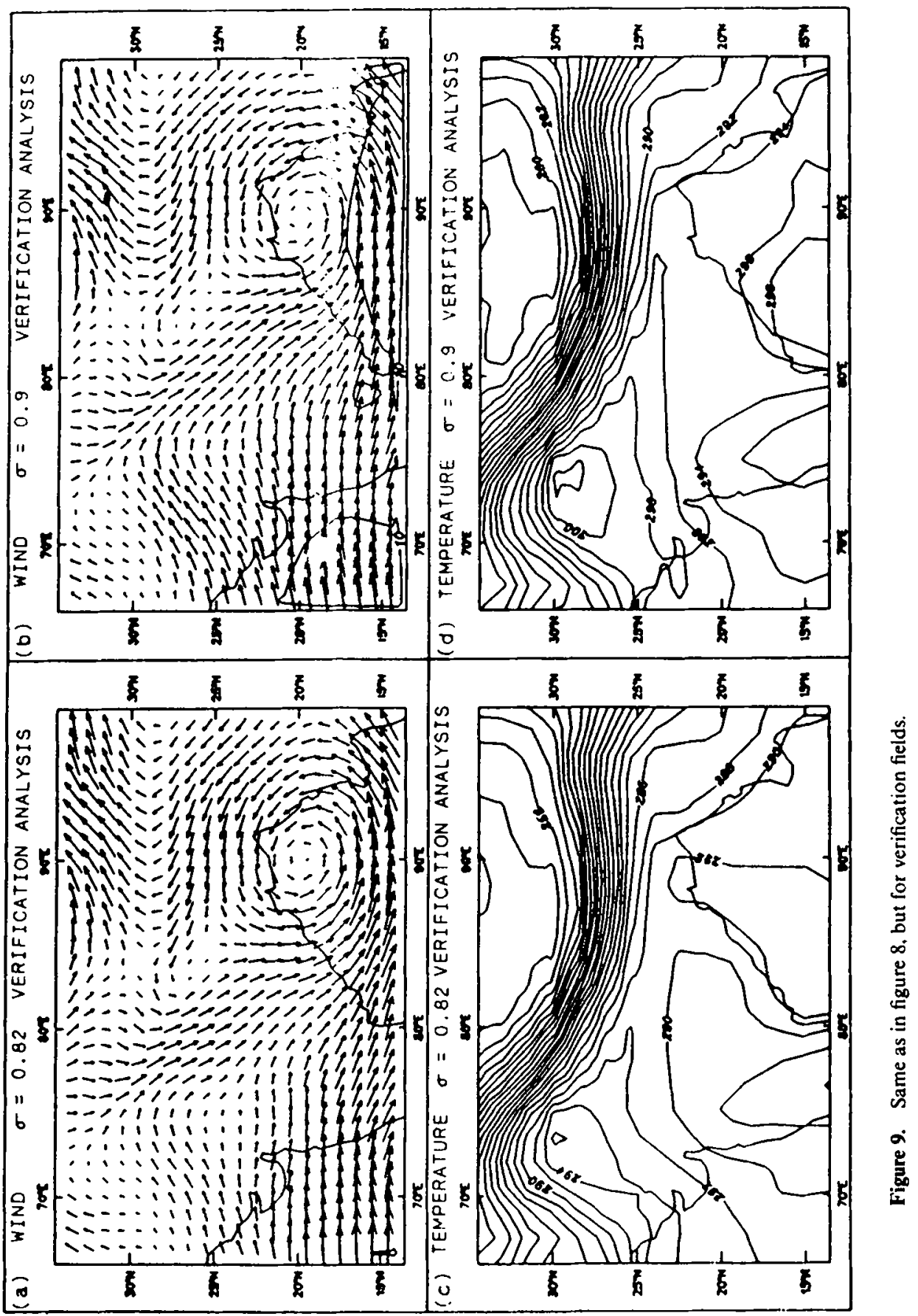


latitude eastward), the zone of confluence in the model generated forecasts (figure 8b) is found to be stronger than the verifying analysis. In particular, enhanced westerlies over the peninsular India in the forecasts added to the building up of intense moisture flux convergence surrounding the vortex. But at $0.82 \sigma$ level, the wind flow predicted over south Madhya Pradesh and the adjoining region is considerably weaker (figure 8a) when compared to the verifying analysis (ngure 9a). However the circulation around the vortex including its center compares well with its verification pattern.

Temperature prediction (figure $8 c$ and $d$ ) and corresponding verification fields (figures $9 \mathrm{c}$ and $\mathrm{d}$ ) show that temperature has been predicted reasonably well by the model. The precipitation field for 24 hours (figure 10a) as predicted by the model agrees
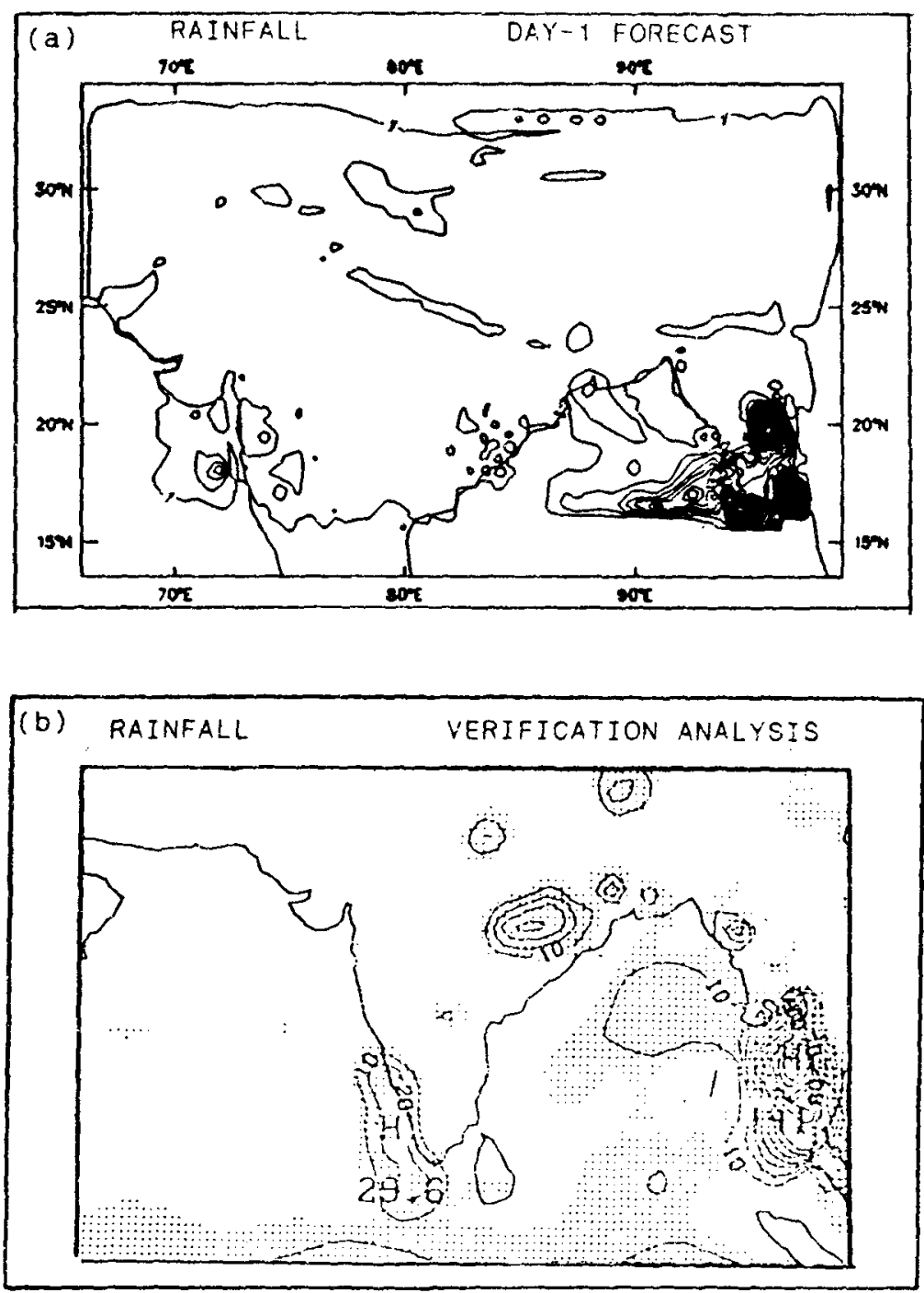

Figure 10. (a) 24 hour forecast of rainfall ( $\mathrm{mm}$ ); (b) Analysis of 24 hour rainfall ( $\mathrm{mm}$ ) ending $00 Z$ 6th July 1979. 
reasonably with the analysis of precipitation field (Krishnamurti et al 1983) depicted in figure $10(\mathrm{~b})$. The maximum precipitation field predicted by the model is $74 \mathrm{~mm}$ near the Arakan coast.

\section{Conclusions}

The following conclusions are drawn from this study of PBL characteristics over the monsoon trough region:

- Diurnal variation of TKE is a marked feature over the land compared to ocean. TKE increases from the eastern side of the monsoon trough to the western sector. The maximum magnitude of TKE over land is of the order of $1-1.5 \mathrm{~m}^{2} / \mathrm{s}^{2}$ over the desert sector of India.

- Presence of low level wind maxima, which is a characteristic feature of turbulent boundary layer, is well simulated by this improved boundary layer physics.

- The model has been able to predict the large scale fields and the evolution of PBL reasonably well in spite of its limitations of not having radiation parameterization scheme and sophisticated energy balance equation to predict the ground temperature. It may be stated in this regard that for short range simulations (up to 1 day) radiation does not play a very important role.

\section{Acknowledgements}

We are thankful to Dr. R V Madala, NRL, Washington DC and Prof. Sethuraman, NCSU, Raleigh, USA for providing an idealized version of the NRL model to our group and for the continued co-operation. We wish to thank NCMRWF, New Delhi for providing the necessary computer facilities. We express our thanks to ECMWF, Reading, UK, for providing the FGGE level III analyses. This work was supported in part by the Office of Naval Research, U.S.A. and the Department of Science and Technology, New Delhi, India.

\section{References}

Anthes R A and Chang S W 1978 Response of the hurricane boundary layer to changes of sea surface temperature; J. Atmos. Sci. 35 1240- 1255

Businger J A, Wyngaard J C. Izumi Y and Bradley E F 1971 Flux-profile relationship in the atmospheric surface layer; J. Atmos. Sci. $28181-189$

Chang S W 1979 An efficient parameterization of convective and non-convective planetary boundary layers for use in numerical models; J. Appl. Meteorol. 1812051215

Daly B J and Harlow F H 1970 Transport equations in turbulence; Phys. Fluids 13 2634-2649

Deardorff J W 1966 The counter gradient heat flux in the lower atmosphere and in the laboratory; J. Atmos. Sci. 23 503-506

Deardorff J W 1974b Three dimensional numerical study of turbulence in an entraining mixed layer; Boundary-Layer Meteorol. $7199-226$

Detering $H W$ and Etling D 1985 A pplication of the $E-\varepsilon$ turbulence model to the atmospheric boundary layer: Boundary-Layer Meteorol. 33 113-133

Gray W M 1975 Tropical cyclone genesis: Atmos. Sci. No. 234 (Colorado State University) 119p 
Holt T and Sethuraman $1988 \mathrm{~A}$ review and Comparative Evaluation of Multi level Boundary Layer Parameterizations for First-order and Turbulent Kinetic Energy Closure Schemes; Rev. Geophys. 26 $761-780$

Krishnamurti T N, Cocke S, Pasch R and Low-Nam S 1983 Precipitation estimates from rain gauge and Satellite observations - Summer MONEX; FSU Report No. 83-7, May 1983, Florida State University, Tallahasse, Florida 373pp.

Krishnamurti T N, Oosterhot D and Nancy Dignon 1989 Hurricane prediction with a high resolution global model; Mon. Weather. Rev. 117 631-669

Kuo H L 1974 Further studies of the parameterization of the influence on cumulus convection on large scale flow; J. Atmos. Sci. 31 1232-1240

Madala R V, Chang S W, Mohanty U C, Madan S C, Paliwal R K, Sarin V B, Holt T and Sethuraman 1987 Description of Naval Research Laboratory Limited Area Dynamical Weather Prediction Model; N R L Tech. Report 5992, Washington D C 131 pp

Mailhot J and Benoit R 1982 A finite element model of the atmospheric boundary layer suitable for use with numerical weather prediction; J. Atmos. Sci. 39 2249-2266

McBean G A K, Bernhardt S, Bodin Z, Litynska A P, Van Ulden and Wyngaard J C 1979, The Planetary Boundary Layer; WMO Tech Note 165 World. Met. Org.

Miller B I 1958 On the maximum intensity of hurricanes; J. Meteorol. 15 184-195

Mohanty U C, Paliwal R K, Madan S C, Tyagi A, Sarin V B and Ramesh K J 1987 Description of a limited area numerical weather prediction model for India and its neighbourhood; Centre for Atmospheric Sciences, IIT, Delhi.

Monin A S and Yaglom A M 1971 Statistical Fluid Mechanics; Vol I MIT Press 468-504

Namias J 1973 Birth of hurricane Agnes-triggered by the trans-equatorial movement of a mesoscale system into a favourable large scale environment; Mon. Weather Rev. 101 177-179

Palmen E 1948 On the formation and structure of tropical hurricanes; Geophysics 3 26-38

Perkey D J and Kreitzberg W 1976 A time dependent lateral boundary scheme for limited area primitive equation model;.Mon. Weather Rev. 104 744-755

Seller W D 1965 Physical Climatology; (University of Chicago Press) 53-54

Shir C C 1973 A preliminary numerical study of atmospheric turbulent flows in the idealized planetary boundary layer; J. Atmos. Sci. $301327-1339$

Tisdale C F and Clapp P F 1963 Origin and paths of hurricanes and tropical storms related to certain physical parameters at the air-sea surface; J. Appl. Meteorol. 2 358-367

Tyagi A, Mohanty U C and Ramesh K J 1994 Structure of the atmospheric boundary layer over the monsoon trough region; Mausam (In press)

Wyngaard J C 1975 Modeling the Planetary boundary layer -- extension to the stable case; Boundary-Layer Meteorol. $9441-460$ 